\title{
ZUCHTWERTSCHATZUNG VON VATERTIEREN UNTER EXAKTER Gewichtung der Nachkommendurchschnitte je Mutter
}

\section{W. KüHne, - Lohmann Tierzucht GmbH Am Seedeich 9, 2190 Cuxhaven, Deutschland.}

Zur Verminderung des Rechenaufwandes bei Zuchtwertschätzungen werden oftmals vereinfachende Annahmen über die zwischen den Informanten bestehenden Verwandtschaftsverhältnisse getroffen. Es wurde anhand eines Beispiels gezeigt, welchen Einfluß eine Fehleinschätzung eines Beispiels gezeigt, welchen Einfluß eine Fehleinschätzung der Familienstruktur auf die Genauigkeit der Regressionskoeffizienten, sowie der multiplen Korrelationskoeffizienten - zwischen den Informationsquellen und dem Zuchtwert - haben. Je größer $h^{2}$ und $c^{2}$ werden, desto größer sind die Fehler, die bei der Errechnung der Regressionskoeffizienten gemacht werden, wenn die angenommene Familienstruktur nicht der tatsächlichen entspricht.

\section{Einfluss DER KontrollhäUfigkeit AUF DEN NetToselektionserfolg Beim ZWEINUTZUNGSRIND}

\section{Jongeling. - Institute of Animal Breeding and Genetics University of Göttingen, 34 Göttingen, BRD.}

Aufgrund ihres hohen Personalkostenanteils ist die Milchkontrolle einem permanenten Kostendruck ausgesetzt. Deshalb sollte die Einführung kostengünstiger Formen der Milchleistungsprüfung in Erwägung gezogen werden. Am Beispiel der Verlängerung der Kontrollintervalle werden im vorliegenden Bericht die züchterischen Konsequenzen studiert, deren Ergebnisse sich folgendermaßen zusammenfassen lassen :

I. Eine Ausdehnung der Prüfungszeiträume kann in Einzelfällen eine beträchtliche Fehleinschätzung der Leistung zur Folge haben. Als wesentliche Fehlerquellen müssen die Länge des ersten und letzten Kontrollintervalls sowie der Laktationsmonat angesehen werden, in dem die Kontrolle einsetzt.

2. Durch diese Kontrollfehler bei 2-monatlicher und 3-monatlicher Probenahme wird die Heritabilität für Fettmenge um 6 bzw. is p. Ioo verringert. Dieser Genauigkeitsverlust kann bezüglich der Bullen durch eine umfangreichere Nachkommenprüfung kompensiert werden.

3. Die Verlängerung der Kontrollintervalle führt zu einer geringfügigen Reduktion des genetischen Fortschritts in Fettmenge.

4. Da aber andererseits beträchtliche Kosteneinsparungen möglich sind, erhöht sich der Nettoselektionserfolg gegenüber der Standardkontrolle.

5. Der Prüfungszeitraum kann bis zu 3 Monaten ausgedehnt werden, ohne daß die optimale Konstellation von Rinderzuchtprogrammen verschoben wird.

Für die Zuchtplanung ergibt sich daraus eine durchaus positive Beurteilung einer derartigen Vereinfachung der Milchleistungsprüfung. Dem größeren Risiko bei der Bullenmütterselektion kann durch die Berücksichtigung des Zuchtwertes des Vaters sowie durch eine Vorsolektion mit nachfolgender genauerer Prüfung begegnet werden.

\section{THEORETICAL Distribution OF FOREIGN GENES IN DIFFERENT CROSSBRED GENERATIONS}

\section{A. EssL. - Institut für Tierproduktion der Universilät für Bodenkultur, Wien}

Probability rules are used to develop formulae for computing distributions of foreign chromosomes (no crossing over) in generations $F_{1}, F_{2}, B C 1, B C 2, B C ~ 3$ ans in the cross $B C$ I $\times F_{1}(B C=$ backcross $)$. Distributions applicable to cattle $(2 n=60)$ and for $2 n=10$ are shown in diagrams and results are discussed. 